\title{
Errata: Multimodal biometric method that combines veins, prints, and shape of a finger
}

\section{Byung Jun Kang}

Technical Research Institute

Hyundai Mobis

80-9 Mabook-dong

Giheung-gu, Yongin-shi

Gyunggi-do 446-912

Republic of Korea

\section{Kang Ryoung Park}

Dongguk University

Division of Electronics and Electrical Engineering

26, Pil-dong 3-ga

Jung-gu, Seoul 100-715

Republic of Korea

E-mail: parkgr@dongguk.edu

\author{
Jang-Hee Yoo \\ Jeong Nyeo Kim \\ Electronics and Telecommunications Research \\ Institute \\ 138 Gajeongno Yuseong-gu \\ Daejeon, 305-700 \\ Republic of Korea
}

[DOI: 10.1117/1.3555176]

This article [Opt. Eng. 50, 017201 (2011)] was originally published on 24 January 2011 with an error in Fig. 1. The correct image appears below.

The corrected manuscript was published online 26 January 2010. The article appears correctly in print.

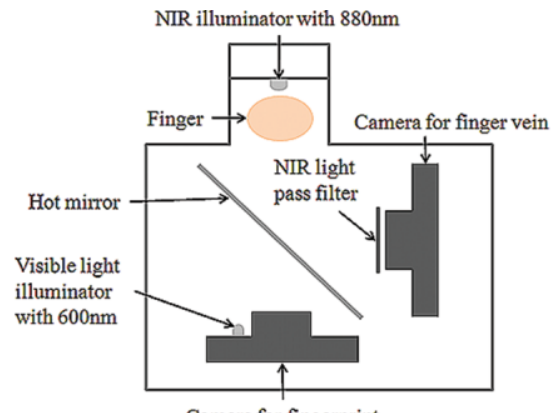

Camera for fingerprint

(a)

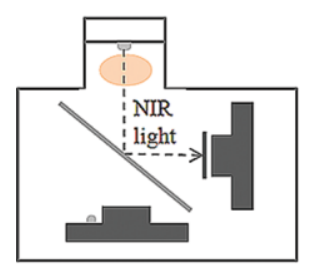

(b)

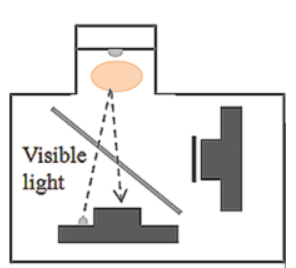

(c)

Fig. 1 